\title{
The Implementation of a Digital Filter-Based Algorithm for Active Power Measurement using an ARM Microcontroller
}

\author{
Ajakida Eski, Orion Zavalani, Denald Komici
}

\begin{abstract}
Since in Albania are still present the electromechanical meters, an urgent need to control the electricity consumption with an efficient and reliable system requires the use of the most modern measurement techniques and the realization of energy measurement devices with more efficiency and precision. This paper provides a software implementation of the real power measurement using an ARM Cortex MO, PSoC4 microcontroller which is responsible for executing algorithms required for the calculation and ensures high performances and low cost. A software based Average Active Power Converter is presented to calculate the real power from the voltage and current signals. This paper briefly covers the mathematics of active power and offers a measurement algorithm based in IIR digital filter, which is more versatile when compared to the analogue filters in their characteristics such as programming flexibility, ability to handle both low as well as high frequency signals accurately. To check the performance of the proposed measurement system, the calculated active power from the standard values of voltage and current is compared to the measured active power from our system and is concluded that the meter has been successfully completed.
\end{abstract}

Index Terms - Algorithm; Digital Filter; Active Power.

\section{INTRODUCTION}

Still in Albania, the energy sector faces a serious problem of revenue collection for supplied electricity due to power theft and network losses. One of the main reasons is the current measurement and billing system, in which electromechanical meters are still present. This system has high cost of measurement because it needs a great staff, low accuracy of readings, difficulties in monitoring ratings, and creates opportunities for abuses. The urgent need to control consumer electricity consumption should be based on an efficient and reliable system for accurate readings, which reduces the cost of measurements, provides a safe environment for measurements and calculations, thus increasing network efficiency, reducing consumption and increasing the value of financial benefits.

The technological developments of recent years create opportunities for using the most modern measurement techniques and the realization of energy measurement devices with more efficiency and precision. There is no doubt that the future lies in automated computer-based metering systems where metering data is read and processed automatically, and all measurement, monitoring and control issues are resolved in real time.
Energy measurement is the process of calculating the power and energy from current and voltage, because the energy is equivalent to the power consumption over time [1]. Energy meters work by constantly measuring the instantaneous voltage and current, the product of which provides instantaneous power (watts), which then integrates with time to evaluate the energy used.

Real power measurement is the process of calculating it through measured instantaneous current and voltage values. This process can be either in software or hardware [2]. There are many integrated dedicated circuits that perform real power measurement in hardware. This paper provides a software implementation of real power measurement.

We calculate the active power $(P)$ using the average power converter which provides an algorithm based on digital filter. Digital filters can achieve thousands of times better performance than analog filters [3]. Digital filters are more versatile when compared to analog filters in their features, such as programming flexibility, ability to handle both low as well as high frequency signals accurately.

The proposed measurement system is based on a single low-cost microcontroller for sampling and digital processing of the input signals. The ARM Cortex MO, PSoC4, CY8C4245AXI-483 microcontroller is responsible for executing algorithms required for the calculation.

\section{DESCRIPTION OF ALGORITHM FOR MEASURING OF THE RMS VALUES}

The power is defined as the rate of energy flow from source to load and it is calculated as the product of the instantaneous values of the current and voltage signal. The resulting signal is called the instantaneous power signal and is equal to the rate of energy flow at every instant of time and the measuring unit is the watt or joules/sec. In an AC system with current and voltage signal

$v(t)=\sqrt{2} \cdot V \sin (\omega t)$

$i(t)=\sqrt{2} \cdot I \sin (\omega t)$

where $\mathrm{V}$ is the RMS voltage and I is the RMS current, the instantaneous power signal is

$$
\begin{aligned}
& p(t)=v(t) \cdot i(t) \\
& p(t)=V I-V I \cos (2 \omega t)
\end{aligned}
$$


Mathematically, the average value of a periodic function is defined as the integral of the function over a full period divided by the period. Therefore, the average instantaneous power value $p(t)$ is given by expression

$P=\frac{1}{T_{1}} \int_{0}^{T_{1}} p(t) d t$

where $T_{1}$ is the period of the signal $p(t)$ and $P$ is the active or real power.

If we integrate over an integer of signal period, let say $n T_{1}$ (where $n$ is a positive integer), then the full surface is calculated by multiplying $n$ with the surface defined by the integral (2). So, we have

$P=\frac{1}{n T_{1}} \int_{0}^{n T_{1}} p(t) d t$

If we choose such that $T=n T_{1}$ ( $T$ is the period of the alternating current and voltage signal) then

$P=\frac{1}{T} \int_{0}^{T} p(t) d t$

Then, we can say that the average power can be calculated by integrating over the period of $\mathrm{i}(\mathrm{t})$ or $\mathrm{v}(\mathrm{t})$ signals. In fact, the average power of any number of complete cycles is the same as the average power value of just one cycle. Thus, we can easily find the average power of a periodic signal, simply by calculating the average value of one complete cycle. Emphasize that the active or real power is equal to the DC component of the instantaneous power signal $p(t)$ in equation (1), i.e., $V I$.

This relationship (3) is used to calculate active power. We calculate active power using average power converter [4] which is the same to the explicit RMS converter. The instantaneous power signal $p(t)$ is generated by multiplying the instantaneous samples of phase voltage signal and phase current signal. The DC component of the instantaneous power signal (active power) is extracted by filtering the instantaneous power signal with a two-stage Butterworth low-pass filter, as shown in figure 1.

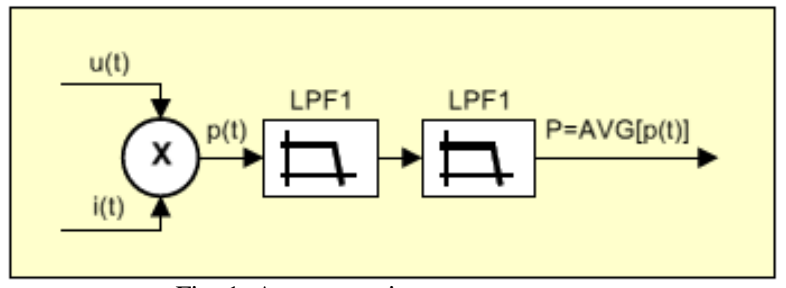

Fig. 1. Average active power converter

This algorithm uses an IIR filter (Infinite Impulse Response). Impulse response functions of IIR filters are nonzero over an infinite length of time. IIR filters use a recursive algorithm, in which the result of one calculation is used in future calculations. Since the output values of IIR filters depend on the previous values, IIR filters has feedback and are recursive.

The advantage of IIR filters [5] over FIR filters is that IIR filters usually require fewer coefficients to execute similar filtering operations, that IIR filters work faster, and require less memory space.
The disadvantage of IIR filters is the nonlinear phase response. IIR filters are well suited for applications that require no phase information, for example, for monitoring the signal amplitudes. The cutting frequency of this filter is selected $3 \mathrm{~Hz}$.

\section{HARDWARE CONSIDERATIONS}

Figure 2 shows the conceptual design of the proposed measurement system. This system is based on a single low cost ARM Cortex MO, PSoC4 microcontroller which cannot make direct measurement [6] when supply voltage is $230 \mathrm{~V}$ at up to $60 \mathrm{~A}$. Therefore, it is necessary to indirectly measure phase voltage and current at a level consistent with a microcontroller. Then rescale these measurements to arrive at the original value. To measure voltage, the phase voltage is scaled down by a potential divider and then passed through an anti-aliasing filter. For current measurement, a current transformer creates voltage signals across two burden resistors that are proportional to the load current and then passed through anti-aliasing filters. These conditioned signals are fed to the ADC channels of ARM Cortex MO, PSoC4.

The A/D Converter of microcontroller performs the digital conversion of both input signals. The bandwidth and accuracy of measurement system are the two main specifications. The bandwidth of the system is decided by the sampling frequency while accuracy depends on resolution of the A/D Converter. The ADC's sample rate should be selected to be sufficient to meet the Nyquist rate [7] for the type of measurement that will make (i.e., slightly greater than twice the highest frequency that is desired to be measured), otherwise, the high-frequency content will alias at a frequency inside the spectrum of interest (pass band). For measurements with a certain accuracy over a given range, the resolution (effective number of bits) of the A/D Converter is calculated. For achieving the accuracy of the order $1 \%$, which is compliant with EN50470-1 and EN50470-3 Europeans standards for electronic meters of active energy classes B, A/D converter with 12 bit resolution [8] and sampling rate of 3200 sample/second is sufficient.

From the digitized values of current and voltage is calculated active power. The ARM Cortex MO, PSoC4, CY8C4245AXI-483 microcontroller is responsible for executing algorithms required for calculation of active power value.

ARM microcontroller of system communicates with the next level equipment as a monitoring PC via the Serial interface RS-232. Also, the active power is displayed on $16 \times 2$ LCD.

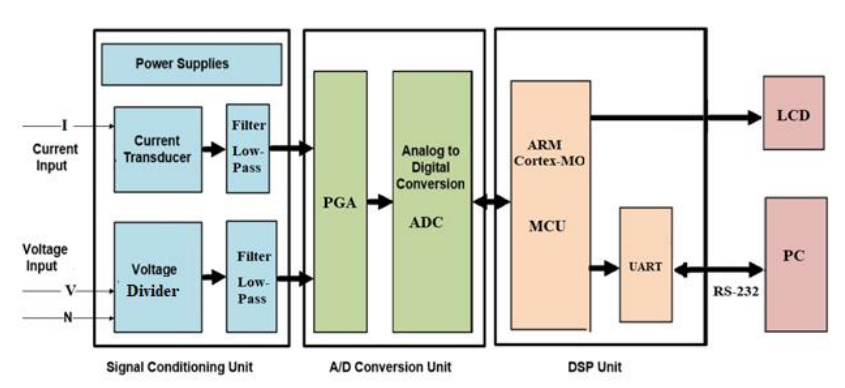

Fig. 2. Block diagram of proposed measurement system 


\section{TESTING, RESUlTS AND DisCUSSION}

The measurement system performance is evaluated in the laboratory. Fig. 3 shows the implementation of laboratory testing.

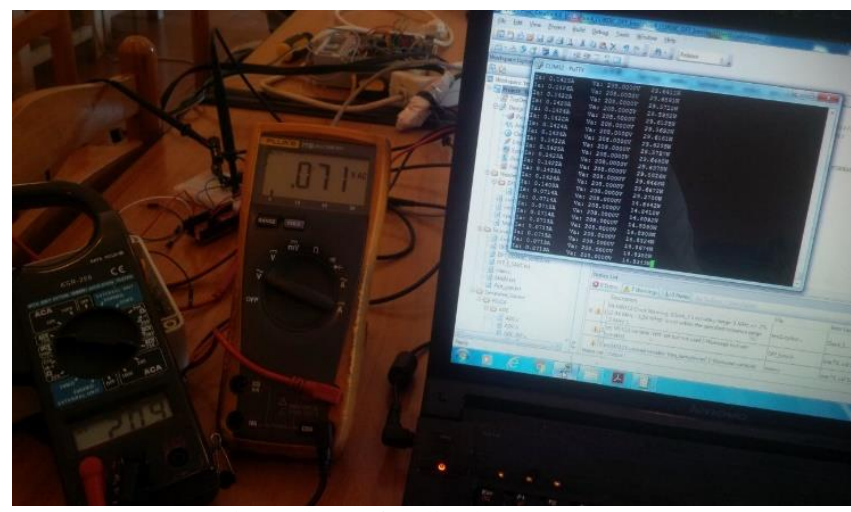

Fig.3. Photo of realized measurements

A current signal is generated with an AFG3021B signal generator. The current and voltage values are measured with standard FLUKE 77III meters. The active power value is calculated from the measured values of current and voltage which are considered as true values.

\section{TABLE I: RESULTS OF MEASUREMENTS IN THE LABORATORY}

\begin{tabular}{|c|c|c|c|c|}
\hline \multirow{2}{*}{$\begin{array}{l}\text { FLUKE } \\
77 \mathrm{III} \\
\text { Voltage } \\
\text { (V) }\end{array}$} & \multirow{2}{*}{$\begin{array}{l}\text { AFG3021B } \\
\text { Function } \\
\text { generator } \\
\text { Current } \\
\text { (A) }\end{array}$} & \multicolumn{2}{|c|}{$\begin{array}{l}\text { Active Power } \\
\text { (W) }\end{array}$} & \multirow{2}{*}{$\begin{array}{c}\text { Relative } \\
\text { error } \\
(\%)\end{array}$} \\
\hline & & $\begin{array}{l}\text { Standard } \\
\text { Meter }\end{array}$ & $\begin{array}{l}\text { Proposed } \\
\text { System }\end{array}$ & \\
\hline 209 & 0.644 & 134.596 & 133.4470 & $-0,85 \%$ \\
\hline 209 & 0.572 & 119.548 & 118.5674 & $-0,82 \%$ \\
\hline 209 & 0.429 & 89.661 & 88.8800 & $-0,87 \%$ \\
\hline
\end{tabular}

To evaluate the performance of the proposed measurement system, the calculated active power from the standard values of voltage and current are compared to the measured active power from our system. The measurements are repeated for different values of current (different load). From the measured values has been calculated the relative error of measurement

Table 1 shows the measured active power values with the standard meter and the proposed system as well as the calculated values of the relative measurement error.

\section{CONCLUSION}

In this paper, we have presented the active power value measurement executing a digital filter based algorithm in an ARM Cortex-MO microcontroller. We have selected an A/D converter with 12 bit resolution and sampling rate of 3200 sample/second so that the measurement system meets the specified bandwidth and accuracy. The calculated error values presented in Table 1 meet the required measurement accuracy by demonstrating that the proposed measurement system has been successfully developed. Since the proposed approach provides low cost solution for true active power measurement, it can be used in industrial and commercial applications.

\section{REFERENCES}

[1] Charles K. Alexander, Matthew N.O. Sadiku, "Fundamentals of Electric Circuits"

[2] Ronald J. Tocci, Neal S. Widmer, Digital System, Principles and Applications", Eighth Edition, ISBN 0-13-085634-7, 2001.

[3] Steven W. Smith, Digital Signal Processing, Second Edition, 1999

[4] Martin Mienkina, "Filter-Based Algorithm for Metering Applications", AN4265, Rev.4, 04/2016.

[5] Rui Guo, "Efficient Hardware Implementation Techniques for Digital Filters", Summer 2011.

[6] S. Tumanski, "Principles of Electrical Measurement" Taylor \& Francis Group, ISBN 0-7503-1038-3, 2006.

[7] Taylor, Fred J., Digital filters: principles and applications with MATLAB.-(IEEE series on digital \& mobile communication; 30) ISBN 978-0-470-77039-9,2012

[8] P.Petrovic, "New Digital Multimeter for Accurate Measurement of Synchronously Sampled AC Signals," IEEE Trans. Instrum.Meas., vol. 53, pp. 716-725, June 2004 